\title{
Crystal Growth in the Presence of Surface Melting: Supersaturation Dependence of the Growth of Columnar Ice Crystals
}

\author{
K. G. LibBrecht AND H. Yu ${ }^{1}$ \\ Norman Bridge Laboratory of Physics, California Institute of Technology 264-33, \\ Pasadena, CA 91125
}

[submitted to the Journal of Crystal Growth, July 31, 2000]

\begin{abstract}
We report measurements of the growth rates of the basal (0001) and prism (10̄10) facets of ice as a function of surface supersaturation, in a regime where the growth dynamics are dominated by the presence of surface melting. Our measurements were made using freely falling columnar ice crystals, growing in a solvent gas of air at a pressure of one atmosphere, with ambient supersaturation levels $1<\sigma_{\infty}<11$ percent. Condensation coefficients for the two facets were inferred by modeling the effects of both particle and heat diffusion, which also yielded surface supersaturation levels $0.75<\sigma_{\text {surf }}<2.2$ percent. Our results show a strong supersaturation dependence in the crystal growth, as for both facets the condensation coefficient exhibited a precipitous drop below $\sigma_{\text {surf }} \approx 1$ percent. In addition, the condensation coefficient for the basal facet was found to be nearly a factor of 10 larger than for the prism facet. These results support a model in which the ice growth rates are governed by $2 \mathrm{D}$ nucleation at the interface between the crystalline solid and a quasi-liquid surface layer.
\end{abstract}

PACS: $81.10 . \mathrm{Bk}, 92.40 . \mathrm{Rm}, 92.60 . \mathrm{Nr}$

Keywords: crystal growth kinetics, ice crystal growth

\section{Introduction}

Many solids exhibit surface melting near the bulk melting temperature, and it is widely believed that the dynamics of crystal growth from the vapor phase is strongly influenced by the presence of surface melting [1-5]. Our theoretical understanding of crystal growth mechanisms in such cases is quite poor, however, owing to the complex nature of the disordered "quasi-liquid" layer (QLL) at the solid/vapor interface [6]. Ice provides an excellent system in which to investigate the effects of surface melting on crystal growth, since there is evidence that a QLL exists on both the prism and basal facets for temperatures above $T \approx-10 \mathrm{C}$ [7]. In addition, for these same temperatures the crystal growth forms are nearly always strongly faceted, indicating the absence of surface roughening (exceptions include the prism facets for $T \gtrsim-2 \mathrm{C}$, and very high supersaturations which produce kinetic roughening [8]). Ice is, in addition, an extremely well studied substance, and many aspects of the growth and surface structure of ice have been characterized using a host of experimental and theoretical techniques.

It is believed that surface melting is the dominant factor giving rise to the dramatic variation of ice crystal growth morphology with temperature: growth forms are plate-like at $-2 \mathrm{C}$, columnar near $-5 \mathrm{C}$, plate-like again at $-15 \mathrm{C}$, and columnar near -30 C [8]. The vapor/quasi-liquid/solid (V/Q/S) model developed by Kuroda, Lacmann and collaborators $[3-5,8]$ proposes that the temperature dependence of the quasi-liquid layer thickness, which is different for the basal and prism facets, affects crystal growth rates in such a way as to produce the observed temperature-dependent growth morphologies. This intriguing, but speculative, model makes several predictions for the growth rates of ice as a function of surface supersaturation which to date have been largely untested. The aim of the present investigation is to examine the $\mathrm{V} / \mathrm{Q} / \mathrm{S}$ model using quantitative measurements of the condensation coefficients as a function of supersaturation.

\footnotetext{
${ }^{1}$ Address correspondence to $k g l @$ caltech.edu (http://www.its.caltech.edu/ atomic/).
} 
At $T=-5.5 \mathrm{C}$, where the present measurements were obtained, the QLL thickness at the ice/vapor interface is approximately $20 \mathrm{~nm}$ thick, and models of surface melting often consider effectively distinct solid/QLL and QLL/vapor interfaces [7]. In this case we can calculate the growth rates at the two interfaces separately, subsequently setting them equal to one another for the actual growth rate. Following Kuroda and Lacmann [3], the growth velocity at the QLL/vapor interface is given by the Hertz-Knudsen equation

$$
v_{Q L L / \text { vapor }}=\alpha_{Q L L} \frac{\Omega\left[p_{\text {surf }}-p(\delta)\right]}{\sqrt{2 \pi m k T}}
$$

where $\alpha_{Q L L}$ is the condensation coefficient of the QLL/vapor interface, $\Omega$ is the molecular volume in the QLL, $\delta$ is the QLL thickness, $p_{\text {surf }}$ is the vapor pressure just above the surface, and $p(\delta)$ is the quasi-equilibrium vapor pressure above a QLL of thickness $\delta$ (defined as the vapor pressure for which the growth rate goes to zero). At equilibrium $\delta=\delta_{0}$ and $p\left(\delta_{0}\right)=p_{0}$, the true equilibrium vapor pressure of the ice surface, while for a growing crystal $\delta>\delta_{0}$ and $p(\delta)>p_{0}$. We have assumed that the QLL/vapor interface is effectively rough (no nucleation barriers), and for further discussion here we will assume that $\alpha_{Q L L}=1$. This latter assumption appears to be a reasonable one, given the long history of measurements of the growth of liquid water droplets, which indicate a condensation coefficient that is at least close to unity [9] (there remains, however, some contradictory evidence in favor of a small condensation coefficient [10]).

In contrast to the QLL/vapor interface, the solid/QLL interface is not rough, as evidenced by the strongly faceted ice crystal growth forms. For the growth conditions considered here, it is thought that the dislocation density at this interface is low [11], and that the growth is limited primarily by 2D nucleation. Then the growth rate is given by a multinucleation model as [12]

$$
v_{\text {solid } / Q L L}=B\left(\Delta \mu_{\text {solid } / Q L L} / k T\right)^{5 / 6} \exp \left[-\Delta G_{2}^{*} / 3 k T\right]
$$

where $\Delta G_{2}^{*} \approx \pi \kappa_{\text {solid } / Q L L}^{2} f_{0} / \Delta \mu_{\text {solid } / Q L L}$ is the formation free energy of a $2 \mathrm{D}$ nucleus, $\kappa_{\text {solid } / Q L L}$ is the edge free energy at the solid/QLL interface, $f_{0}$ is the area occupied by a molecule, and $\Delta \mu_{\text {solid } / Q L L}(\delta)$ is the chemical potential difference at the solid/QLL interface.

The growth dynamics contained in the prefactor $B$ are not well understood, owing to the complexity of the solid/QLL interface. If we assume that the growth is similar to that of a normal solid/liquid interface, we can write $[3,5] B \approx \eta D_{Q L L} / h$, where $D_{Q L L}$ is the diffusion constant in the QLL, $h$ is the step height, and $\eta$ is a phenomenological factor which relates $D_{Q L L}$ to interfacial transport [13]. One expects $D_{Q L L}$ to be considerably smaller than the diffusion constant in the bulk liquid, as has been observed in measurements of rare-gas crystal growth [14].

We define the overall condensation coefficient $\alpha$ by

$$
\begin{aligned}
v & =\alpha \frac{\Omega\left[p_{\text {surf }}-p_{0}\right]}{\sqrt{2 \pi m k T}} \\
& =\alpha \frac{\Omega p_{0} \sigma_{\text {surf }}}{\sqrt{2 \pi m k T}}
\end{aligned}
$$

where $v=v_{Q L L / v a p o r}=v_{\text {solid } / Q L L}$ is the measured surface growth velocity and $\sigma_{\text {surf }} \equiv\left(p_{\text {surf }}-\right.$ $\left.p_{0}\right) / p_{0}$. For the growth conditions of our measurements we find $\alpha \ll 1$, which from the above implies that $p(\delta) \approx p_{\text {surf }}$. Thus the chemical potential difference across the QLL/vapor surface, $\Delta \mu_{Q L L / \text { vapor }}=k T \log \left(p_{\text {surf }} / p(\delta)\right)$, is small, so that the chemical potential drop is nearly all at the solid/QLL layer, giving $\Delta \mu_{\text {solid/QLL }} \approx \Delta \mu=k T \log \left(p_{\text {surf }} / p_{0}\right)$. More quantitatively, $\Delta \mu_{Q L L / \text { vapor }} / \Delta \mu \approx \alpha / \alpha_{Q L L} \ll 1$ in the limit $\sigma_{\text {surf }} \ll 1$, which again implies $\Delta \mu_{\text {solid } / Q L L} \approx \Delta \mu$. With this we have

$$
v \approx B(\Delta \mu / k T)^{5 / 6} \exp \left[-\Delta G_{2}^{*} / 3 k T\right]
$$

with $\Delta G_{2}^{*} \approx \pi \kappa_{\text {solid } / Q L L}^{2} f_{0} / \Delta \mu$. Given this model, measurements of crystal growth rates can be used to infer $B$ and $\kappa_{\text {solid } / Q L L}$. A specific prediction of the $\mathrm{V} / \mathrm{Q} / \mathrm{S}$ model is that the threshold value of $p_{\text {surf }}$ (below which growth is strongly inhibited by a $2 \mathrm{D}$ nucleation barrier) will be fairly low, reflecting the fact that $\kappa_{\text {solid } / Q L L}$ is considerably smaller that $\kappa_{\text {solid/vapor }}$, the latter being a theoretical quantity calculated for a surface without surface melting. 


\section{Free-Fall Crystal Growth Measurements}

Figure 1 shows a schematic diagram of our ice crystal growth chamber, which was used to grow freely falling crystals, thus avoiding systematic errors introduced by substrate interactions [15]. The large volume of the chamber facilitated rapid convective mixing, and thus produced a large supersaturated region in the chamber center, far from the chamber walls. Since the convection was strongly turbulent (Reynolds number $\sim 10^{4}$ ), mixing produced nearly isothermal conditions within the tank: the air temperature $1 \mathrm{~cm}$ above the heated water surfaces was measured to be no more than 1-2 degrees above the temperature in the rest of the tank, and the temperature $10 \mathrm{~cm}$ above the water surface was within a few tenths of a degree of the temperature at the tank center. From these and other measurements we confirmed that the ambient temperature throughout most of the central region of the chamber was constant to within a few tenths of a degree. All the data shown here were taken with the tank center temperature equal to -5.5 C $( \pm 0.1 \mathrm{C})$, which is the temperature at which the growth rate of the prism facets is a local minimum $[8,19]$.

The ambient water vapor supersaturation relative to ice, $\sigma_{\infty} \equiv\left(p_{\infty}-p_{0}\right) / p_{0}$, within the chamber necessarily peaked above the heated water reservoirs, and went to zero at the chamber walls, which were covered with frost. Since convective mixing in the chamber was rapid, and the air temperature nearly constant, this implies that there was a large volume in middle of the tank where the supersaturation was nearly constant during our growth measurements. We measured the supersaturation at the geometrical center of the tank, immediately before and after crystal growth data were taken, using the differential technique described in [20]. The volume of air sampled was much less than the tank volume, and care was taken that the supersaturation was not substantially perturbed by the measurement process [20]. The supersaturation profile was not directly measured throughout the tank, and the variation in supersaturation seen by the growing crystals remains a source of possible systematic error in our measurements (see below).

After the chamber was allowed to reach a stable state (several hours), very few droplets or crystals formed inside the chamber without first being intentionally nucleated. A small fleck of dry ice was dropped into the chamber to nucleate a number of ice crystals, which then grew as they drifted and slowly fell through the chamber. The sizes of the larger crystals that fell onto a small observing window at the bottom of the chamber were subsequently recorded as a function of time after the nucleation event. Figure 2 shows typical data from several growth runs, taken 5-10 minutes apart.

At a given time we observed a substantial number of crystals falling onto the window with sizes considerably smaller than those recorded and shown in Figure 2. We believe that these smaller crystals grew near the walls of the growth chamber, or near other falling crystals, where the supersaturation was lower and the growth rates correspondingly smaller. We observed after a series of growth runs, however, that there was clearly an upper bound to the crystal size at each given time. We believe the upper bound was defined by those crystals which, by chance, spent most of their time in relative isolation drifting through the center of the chamber, where the supersaturation was high. Since we expect a large volume in the tank center with nearly constant supersaturation, it is not surprising that a well-defined upper bound was observed. The effects of competition between growing crystals were observed by varying the number of nucleated crystals in a given run. Care was taken to nucleate a small enough number of crystals so that competition did not affect the observed upper bound in crystal size as a function of time.

Observations were made using two different microscope magnifications. A low-magnification view was used to spot falling crystals, and a high-magnification view was used to zoom in on the larger crystals, which could then be accurately measured from a videotape of the growth run. This procedure produced quite consistent results on a given day, during which time the conditions of the tank were stable. The observed crystal morphologies were predominantly simple hexagonal prisms, always with negligible hollowing of the prism facets. The basal facets did exhibit some hollowing for the larger crystals at the higher supersaturations. We rejected crystals with more complex (usually polycrystalline) morphologies. 
Growth data from a series of runs, producing a set of data like those shown in Figure 2, were combined by drawing power-law curves delineating the approximate maximum crystal size as a function of time. Specifically, we approximated this data by the functional form $L(t)=L_{100}(t / \tau)^{\beta}$ with $\tau=100$ seconds, and independent parameters $L_{100}$ and $\beta$ were chosen for each of the two crystal facets. Length measurements are defined such that $L_{c}$ is the length of the columnar prism, and $L_{a}$ is the tip-to-tip width of the prism. Note that a power-law behavior is expected from consideration of simpler growth forms - for example for spherical crystals limited purely by diffusion $R(t) \sim t^{1 / 2}$, and for needle-like growth $L(t) \sim t$ (when $L$ is large compared to the needle diameter). The situation is more complex for the columnar crystals observed, but we found that all our data were well described by power-law fits.

Figure 3 shows the results of our growth measurements at ambient supersaturation levels in the range $1 \leq \sigma_{\infty} \leq 11$ percent, all taken at a temperature of $-5.5 \mathrm{C}$. These measurements can be compared directly with previous free-fall growth measurements obtained at the water vapor supersaturation level only [21-23]. These other data sets were extrapolated to $t=100$ seconds by assuming growth of the form $L(t)=L_{100}(t / \tau)^{\beta}$, with $\beta_{a}=0.46$ and $\beta_{c}=0.83$, as these exponent values were determined by the present measurements. The data sets by Yamashita [21] and Ryan et al. [22] yielded $L_{100}$ values that agreed fairly well with each other and with the present results, as is shown in Figure 3. This good agreement supports our supposition that convection mixes the ambient gas sufficiently to produce a large region of nearly constant supersaturation in the center of our growth chamber.

The data by Takahashi et al. [23] yielded the values of $L_{a, 100}=42 \mu \mathrm{m}$ and $L_{c, 100}=57 \mu \mathrm{m}$, which are substantially different from Yamashita [21], Ryan et al. [22], and the present data. However, the levitation technique used by Takahashi et al. was quite different in comparison to the free-fall measurements used by the other authors, and this technique was clearly not intended or well-suited for observing very small crystals; thus it is perhaps not too surprising that the Takahashi et al. data set yielded inconsistent crystal sizes at $t=100$ seconds.

We combined all our growth data by drawing curves through the parameters in Figure 3, which yielded the curves: $L_{c, 100}\left(\sigma_{\infty}\right)=135\left[\log _{10}\left(1+\sigma_{\infty}\right)\right]^{1.25}, L_{a, 100}\left(\sigma_{\infty}\right)=30\left[\log _{10}\left(1+\sigma_{\infty}\right)\right]^{1.25}$, $\beta_{c}\left(\sigma_{\infty}\right)=0.75 \sigma_{\infty}^{0.06}$, and $\beta_{a}\left(\sigma_{\infty}\right)=0.3 \sigma_{\infty}^{0.25}$, which are plotted with the data in Figure 3 . Here the $L_{100}$ values are in microns, $\sigma_{\infty}$ is the ambient supersaturation in percent, and the functional forms where chosen simply to fit the data well over the range of our measurements $\left(1<\sigma_{\infty}<11\right)$. We then used these functions to infer the growth of ideal crystals at $\sigma_{\infty}=1,2,4,7$, and 11 percent, and these smoothed data points were used in the subsequent analysis.

\section{Modeling Crystal Growth}

The above measurements of crystal size, giving $L_{a}(t)$ and $L_{c}(t)$, provide a good approximation to the ideal case of an isolated, hexagonal ice prism growing in a medium which has an ambient supersaturation $\sigma_{\infty}$ and temperature $T_{\infty}$. From the fit parameters describing $L_{x}\left(t, \sigma_{\infty}\right)$ for the two facets $(x=a, c)$ we can derive the perpendicular surface growth velocities $v_{x}\left(t, \sigma_{\infty}\right)=\left(d L_{x} / d t\right) / 2$ (ignoring a slight geometrical correction for the prism facet). The time dependence here arises because the surface supersaturation $\sigma_{\text {surf }}$ is itself changing with time, as it depends on the size and morphology of the growing crystal. In order to extract quantitative information on surface kinetics from these velocities, we must model the growth process to obtain the surface supersaturation, thus yielding the physically relevant function $v_{x}\left(\sigma_{\text {surf }}\right)$ (or equivalently $\alpha_{x}\left(\sigma_{\text {surf }}\right)$ ) for the two facets.

The growth of an isolated crystal is governed by three physical processes: particle diffusion, heat diffusion, and surface kinetics. Particle diffusion limits the rate at which molecules can reach the growing crystal, and thus reduces the water vapor pressure just above the crystal surface to a value substantially lower than ambient, so $p_{\text {surf }}<p_{\infty}$. Heat diffusion through the ambient gas carries away the latent heat deposited as the crystal grows, and the balance of the inward and outward heat fluxes raises the crystal temperature to a value greater than ambient, $T_{\text {surf }}>T_{\infty}$. The increased 
temperature results in an increased equilibrium vapor pressure $p_{0}$ of the crystal surface relative to that of a non-growing crystal, since $p_{0, \text { surf }} \equiv p_{0}\left(T_{\text {surf }}\right)>p_{0}\left(T_{\infty}\right)$. Both these processes affect the supersaturation just above the crystal surface, $\sigma_{\text {surf }} \equiv\left(p_{\text {surf }}-p_{0, \text { surf }}\right) / p_{0, \text { surf }}$. The intrinsic physics behind particle and heat diffusion is of course well understood, and the conditions under which our data were taken allow a number of simplifications which make it possible to calculate the effects of both these diffusion processes. By modeling these processes we can then infer quantitative information about surface kinetics. We note that since the Reynolds number associated with the velocity of the falling crystal through the medium is quite small $-\operatorname{Re} \approx(0.005 \mathrm{~m} / \mathrm{sec})\left(10^{-4} \mathrm{~m}\right) /(1.5 \times$ $\left.10^{-5} \mathrm{~m}^{2} / \mathrm{sec}\right) \approx 0.03$ - we are justified in neglecting the so-called ventilation effects of this motion on crystal growth [24].

Crystal heating from the deposition of latent heat during growth was modeled by assuming that generated latent heat is carried away solely by conduction, and in this case the temperature rise of a growing crystal can be estimated as

$$
\Delta T \approx \frac{\left(2 v_{a} L_{c}+\varepsilon v_{c} L_{a}\right) L_{a}}{2\left(L_{a} L_{c}\right)^{1 / 2}} \frac{\rho U}{\kappa}
$$

where $v_{x}$ are the measured surface growth velocities of the two facets, $\rho$ is the density of ice, $U$ is the latent heat of condensation, and $\kappa$ is the thermal conductivity of air. The parameter $\varepsilon$ is a correction factor to take into account the observed hollowing of the basal facets. The high thermal conductivity of ice relative to air, together with the small growth velocities, insures that the crystal temperature is essentially constant throughout the solid. Since the quantities on the right side of the above equation are all known or were measured, it was straightforward to calculate $\Delta T$ for a given growing crystal. For our worst case of growth at $\sigma_{\infty}=11$ percent we find $\Delta T \approx 0.5 \mathrm{C}$, which is substantial, while for smaller values of $\sigma_{\infty}$ the temperature correction was correspondingly smaller.

To model particle diffusion we used a cylindrically symmetric Green's function technique [19], which was further extended to include the effects of surface tension. With this method two approximations are used to greatly simplify the diffusion problem, namely cylindrical symmetry (which reduces the dimensionality of the problem), and the slow-growth approximation (for which the diffusion equation reduces to Laplace's equation). Given that our observations show negligible hollowing of the prism facets in all cases, the first approximation mainly introduces a small geometrical correction. The latter approximation is justified by the fact that the diffusion time $\tau \approx \lambda^{2} / D$, where $\lambda$ is a typical crystal size and $D$ is the diffusion constant in air, was much smaller than the crystal growth time in our experiments.

The diffusion equation in this case is $\nabla^{2} \sigma=0$, where $\sigma \equiv\left(p-p_{0}\right) / p_{0}, p$ is the water vapor partial pressure, and $p_{0}$ is evaluated at $T_{\text {surf }}$, which includes the temperature correction above. For this equation we have the mixed boundary condition

with

$$
K \sigma_{\text {surf }}=\frac{p_{0}}{n_{\text {ice }} k T} D(\widehat{n} \cdot \vec{\nabla} \sigma)_{\text {surf }}
$$

$$
K=\alpha \frac{\Omega p_{0}}{\sqrt{2 \pi m k T}}
$$

and the surface growth velocity is $v=K \sigma_{\text {surf } f}$. The functional form of $K(\widehat{\theta})$, where $\widehat{\theta}$ is the angle of the surface normal relative to the crystal axis, was taken to be cusp-like, with deep local minima for growth in the basal and prism directions, thus producing faceted growth [19]. We define $\alpha_{\text {prism }}$ and $\alpha_{\text {basal }}$ to be the values of $\alpha$ at the cusps for the two facets. These quantities dominate the growth rates of faceted crystals, which are insensitive to the width of the cusps [19].

For a solution to the diffusion equation we input the ambient supersaturation $\sigma_{\infty}$, the crystal dimensions, $L_{a}$ and $L_{c}$, and the two condensation coefficients $\alpha_{b a s a l}$ and $\alpha_{\text {prism }}$. From the solution we obtained $\sigma_{\text {surf }}$, and thus the growth velocities of the two facets. The condensation coefficients were adjusted until the derived growth velocities agreed with the observed values. As a check we compared the results of our code with results using a commercial code which could solve cylindrically symmetric heat diffusion problems [25]. The present particle diffusion problem could easily be cast into the heat diffusion format, provided we used the so-called convective boundary conditions (also 
known as boundary conditions of the third kind).

A modeling approach which produced robust results from our data was to interpolate the data taken at a given supersaturation $\sigma_{\infty}$ to that point in time when the crystal size is $L_{c}=50 \mu \mathrm{m}$. In this way we are looking at the supersaturation dependence of the growth of crystals of nearly identical size and shape, which reduces modeling errors. The $L_{a}$ values are known at these times, as are the surface growth velocities. For these fixed- $L_{c}$ crystals the modeling proceeded by choosing condensation coefficients, $\alpha_{\text {basal }}$ and $\alpha_{\text {prism }}$, for which the model reproduced the observed growth velocities, with the results shown in Figure 4. In these calculations we assumed simple right cylindrical crystals, and assumed that the observed crystal growth velocity was equal to the highest velocity calculated along the length of a given facet. The calculation also produced the supersaturation $\sigma_{\text {surf }}$ at the crystal surface as a function of spatial position.

Since our aim was to produce quantitative measurements of the condensation coefficients, the effects of a number of possible systematic errors were explored in our analysis. The effects of hollowing of the basal facets (which was observed in the larger crystals at the higher supersaturation levels) introduced some uncertainty in our inferred $\alpha_{\text {basal }}$, which arose from inherent modeling effects [19], as well as from changes in the temperature correction described above. We investigated these uncertainties by varying the amount of hollowing input into our model. We also explored how possible systematic errors in our ambient supersaturation measurements may have affected our results. Introducing plausible systematic effects like these typically resulted in changes in the scaling of our inferred $\alpha_{\text {basal }}, \alpha_{\text {prism }}$, and $\sigma_{\text {surf }}$, essentially changing the axes in Figure 4 by small multiplicative factors. However, the essential features derived from our measurements, particularly the rapid increase in $\alpha_{\text {basal }}$ and $\alpha_{\text {prism }}$ above a critical supersaturation, were found to be quite robust, insensitive to modeling details and other potential systematic errors.

\section{Discussion}

A first conclusion from our investigation is that growth measurements like those described above are an effective tool for obtaining quantitative measurements of condensation coefficients describing crystal growth. The free-fall technique allows the growth of small, isolated, single crystals, unfettered by the presence of a substrate. By measuring the upper bound of crystal size as a function of time, one greatly reduces the deleterious effects of competition between growing crystals, and effects from the boundary walls of the growth chamber, since these both act to reduce crystal growth. For isolated crystals we find that particle and heat transport can be adequately modeled using a cylindrically symmetric approximation, allowing the condensation coefficients, $\alpha_{\text {basal }}$ and $\alpha_{\text {prism }}$, to be extracted as a function of $\sigma_{\text {surf }}$, the supersaturation just above the growing surface.

A second conclusion from our investigation is that measurements of growth as a function of surface supersaturation provide an excellent test of the V/Q/S model. Our measured values of the condensation coefficients, $\alpha_{\text {basal }}$ and $\alpha_{\text {prism }}$ shown in Figure 4 , clearly show a pronounced increase for $\sigma_{\text {surf }} \gtrsim 1$ percent, a result which proved to be quite insensitive to modeling uncertainties and other plausible potential systematic errors [26]. We attribute this threshold behavior to a 2D nucleation barrier at the solid/QLL interface, as was proposed in the V/Q/S model of Kuroda and Lacmann [3]. Using the multinucleation model described above, we expect that the condensation coefficients can be described by

$$
\begin{aligned}
\alpha_{x}\left(\sigma_{\text {surf }}\right) & \approx B_{x} \frac{\sqrt{2 \pi m k T}}{\Omega p_{0}} \sigma_{\text {surf }}^{-1 / 6} \exp \left[-\Delta G_{2, x}^{*} / 3 k T\right] \\
& \approx B_{x} \frac{\sqrt{2 \pi m k T}}{\Omega p_{0}} \sigma_{\text {surf }}^{-1 / 6} \exp \left[-\pi \kappa_{\text {solid } / Q L L, x}^{2} f_{0, x} / 3 k^{2} T^{2} \sigma_{\text {surf }}\right]
\end{aligned}
$$

where we have used $\sigma_{\text {surf }} \approx \Delta \mu / k T$. Fitting this functional form to our data yields the curves $\alpha_{\text {basal }} \approx 0.1 \sigma_{\text {surf }}^{-1 / 6} \exp \left(-1 / \sigma_{\text {surf }}\right)$ and $\alpha_{\text {prism }} \approx 0.03 \sigma_{\text {surf }}^{-1 / 6} \exp \left(-1.3 / \sigma_{\text {surf }}\right)$, (where $\sigma_{\text {surf }}$ is in percent) as shown in Figure 4 . The threshold behavior in the data is clearly even more pronounced than 
the model. From the fit we find $\kappa_{\text {solid } / Q L L, \text { basal }} \approx 1.1 \times 10^{-12} \mathrm{~J} / \mathrm{m}$ and $\kappa_{\text {solid } / Q L L, \text { prism }} \approx 1.3 \times 10^{-12}$ $\mathrm{J} / \mathrm{m}$. These values are only slightly larger than the value $\kappa_{\text {solid/liquid }} \approx 7 \times 10^{-13} \mathrm{~J} / \mathrm{m}$ derived from measurements of ice growth from the liquid [3], and are considerably smaller than $\kappa_{\text {solid/vapor }} \approx 4 \times 10^{-11} \mathrm{~J} / \mathrm{m}[3]$. This result strongly supports the $\mathrm{V} / \mathrm{Q} / \mathrm{S}$ model, and that a QLL does exist on both the prism and basal faces at $T=-5.5 \mathrm{C}$. Nevertheless, we must acknowledge that the $\mathrm{V} / \mathrm{Q} / \mathrm{S}$ model is not necessarily the correct explanation of our data, since we do not know the QLL thickness at this temperature with any real accuracy.

An interesting feature of the $\mathrm{V} / \mathrm{Q} / \mathrm{S}$ model is that the threshold surface supersaturation (below which the growth is strongly limited by $2 \mathrm{D}$ nucleation) is expected to be approximately equal for the basal and prism facets, since we expect $\kappa_{\text {solid } / Q L L}$ to be only weakly dependent on $\delta$, the QLL thickness [3]. Our measurements support this expectation. From these observations we see that surface melting substantially reduces the 2D nucleation barrier (relative to that of a bare solid/vapor interface), which in turn enhances crystal growth. But in contrast to surface roughening, which completely eliminates the nucleation barrier, for the case of surface melting we see that the location of the 2D nucleation barrier is moved to the solid/QLL interface.

From the fit values of the prefactors $B_{x}$ we find $\left(\eta D_{Q L L}\right)_{\text {basal }} \approx 2.9 \times 10^{-14} \mathrm{~m}^{2} / \mathrm{sec}$ and $\left(\eta D_{Q L L}\right)_{\text {prism }} \approx 4.4 \times 10^{-15} \mathrm{~m}^{2} / \mathrm{sec}$. Assuming $\eta_{x} \approx 1$, we see these values are comparable to $D_{Q L L} \approx 3 \times 10^{-14} \mathrm{~m}^{2} / \mathrm{sec}$ obtained from NMR measurements of polycrystalline ice [5,27]. This also confirms $D_{Q L L} \ll D_{\text {liquid }}$, consistent with the results obtained from measurements of rare gas crystal growth [14]. According to the V/Q/S model, the different values of $\eta D_{Q L L}$ we infer for the two facets reflect the different structures of the two surfaces, since $D_{Q L L}$ is expected to be strongly dependent on the QLL thickness.

The qualitative and quantitative agreement between our measurements and the V/Q/S model is encouraging, although clearly the ice surface structure and growth dynamics are quite complex. We are currently undertaking experiments to systematically measure ice crystal growth rates as a function of supersaturation over a range of different temperatures and in different solvent gases, which we believe will help shed new light on the relationship between surface structure and crystal growth.

\section{Acknowledgments}

H. Y. acknowledges financial support from the Summer Undergraduate Research Fellowship program of the California Institute of Technology.

\section{References}

[1] J. P. van der Eerden, J. Cryst. Growth 128 (1993) 62.

[2] J. F. van der Veen et al., in Chemistry and Physics of Solid Surfaces VII (R. Vanselow and R. F. Howe, eds.) (Springer-Verlag: Berlin) (1988) 455.

[3] T. Kuroda and R. Lacmann, J. Cryst. Growth 56 (1982) 189.

[4] T. Kuroda, J. Meteor. Soc. Japan 60 (1982) 520.

[5] T. Kuroda, J. Cryst. Growth 99 (1990) 83.

[6] J. P. van der Eerden, Faraday Discuss 95 (1993) 65.

[7] Different experimental surface probes, such as glancing incidence X-ray scattering, optical ellipsometry, and proton backscattering, do not give consistent results for the QLL thickness as a function of temperature; these data are reviewed by V. F. Petrenko and R. W. Whitworth, Physics of Ice (Oxford University Press: Oxford) (1999), and J. G. Dash, H. Fu, and J. S. Wettlaufer, Rep. Prog. Phys 58 (1995) 115. There is also some evidence that the QLL thickness depends on the pressure and composition of the background gas to which the surface is exposed. 
[8] Ice crystal growth from vapor is reviewed by T. Kobayashi and T. Kuroda, in Morphology of Crystals, Part B (I. Sunagawa, ed.) (Terra Scientific: Tokyo) (1987).

[9] I. W. Eames, N. J. Marr, and H. Sabir, Int. J. Heat Mass Transfer 40 (1997) 2963.

[10] R. A. Shaw and D. Lamb, J. Chem. Phys. 111 (1999) 10659.

[11] F. C. Frank, Contemp. Phys. 23 (1982) 1; C. V. McKnight and J. Hallett, J. Cryst. Growth 46 (1979) 427.

[12] I. V. Markov, Crystal Growth for Beginners (World Scientific: Singapore) (1995); Y. Saito, Statistical Physics of Crystal Growth (World Scientific: Singapore) (1996).

[13] J. W. Cahn, W. B. Hillig, and G. W. Sears, Acta Met. 12 (1964) 1421; S. D. Peteves and R. Abbaschian, Met. Trans. 22A (1991) 1271.

[14] M. Maruyama, J. Cryst. Growth 94 (1989) 757.

[15] Several quantitative ice growth measurements as a function of supersaturation have been made under pure water vapor conditions, i.e. in the absence of a solvent vapor, by growing crystals on substrates [16-18]. Since the vapor diffusion constant is large in this case, particle transport effects do not significantly hinder crystal growth, and the growth rates are in principal determined mainly by surface kinetics. Unfortunately, measured growth rates which have been reported under ostensibly identical conditions differ markedly from one another. For example, these three papers [16-18] each describe measurements of the growth rates of ice basal and prism facets at -7 $\mathrm{C}$, and each includes data taken at water vapor supersaturation levels near 0.5 percent. Results of the three experiments vary by over a factor of 10 , and the origin of the discrepancies is not clear. One potential systematic error was observed by Beckmann and Lacmann, who found that the growth rates of prism facets could vary by a factor of five on the same crystal, depending on whether the facet was contacting the substrate or not. Lamb and Scott also reported growth anomalies for their substrate-grown crystals.

[16] D. Lamb and W. D. Scott, J. Cryst. Growth 12 (1972) 21.

[17] W. Beckmann and R. Lacmann, J. Cryst. Growth 58 (1982) 433.

[18] T. Sei and T. Gonda, J. Cryst. Growth 94 (1989) 697.

[19] K. G. Libbrecht, Phys. Rev. E 60 (1999) 1967.

[20] C. K. Chng, H. Yu, K. G. Libbrecht, Rev. Sci. Instrum. 71 (2000) 266.

[21] A. Yamashita, Kisho Kenkyu Noto, Met. Soc. Japan 123 (1974) 47 (in Japanese); a discussion of these results, in English, is given in T. Kobayashi and T. Kuroda, in Morphology of Crystals, Part B (I. Sunagawa, ed.) (Terra Scientific: Tokyo) (1987).

[22] B. F. Ryan, E. R. Wishart, and D. E. Shaw, J. Atmos. Sci. 33 (1976) 842.

[23] T. Takahashi, T. Endoh, G. Wakahama, and N. Fukuta, J. Meteor. Soc. Japan 69 (1991) 15.

[24] T. Kuroda, J. Meteor. Soc. Japan 62 (1984) 552.

[25] Quickfield version 4.1, by the Tera Analysis Company, Svendborg, Denmark (1998).

[26] It is noteworthy that the values of $\alpha_{\text {basal }}$ we infer from our present data are substantially lower than what we reported earlier [19], based on the crystal growth data by Yamashita. We found that the origin of this discrepancy was the deep hollowing that occurs for larger crystals like those grown by Yamashita, along with crystal heating effects. Our earlier modeling used a fairly course grid on the crystal surface (to encourage stable growth), which we now realize does not produce sufficient basal hollowing for large crystals. Thus our previous model crystals, with insufficient hollowing, needed a high value of the condensation coefficient to reproduce the Yamashita data. With more realistic hollowing we find that a smaller coefficient is needed. In our present data the crystals are smaller, with much reduced basal hollowing, and are thus much more easily modeled. Inferred values of $\alpha_{\text {prism }}$ from the present data are roughly the same as we reported earlier, since the prism facets exhibit negligible hollowing under these conditions.

[27] Y. Mizuno and N. Hanafusa, in: Proc. 7th Symp. on Physics and Chemistry of Ice, Grenoble, 1986 [J. Physique Colloq. 48 (1987) C1-511]. 


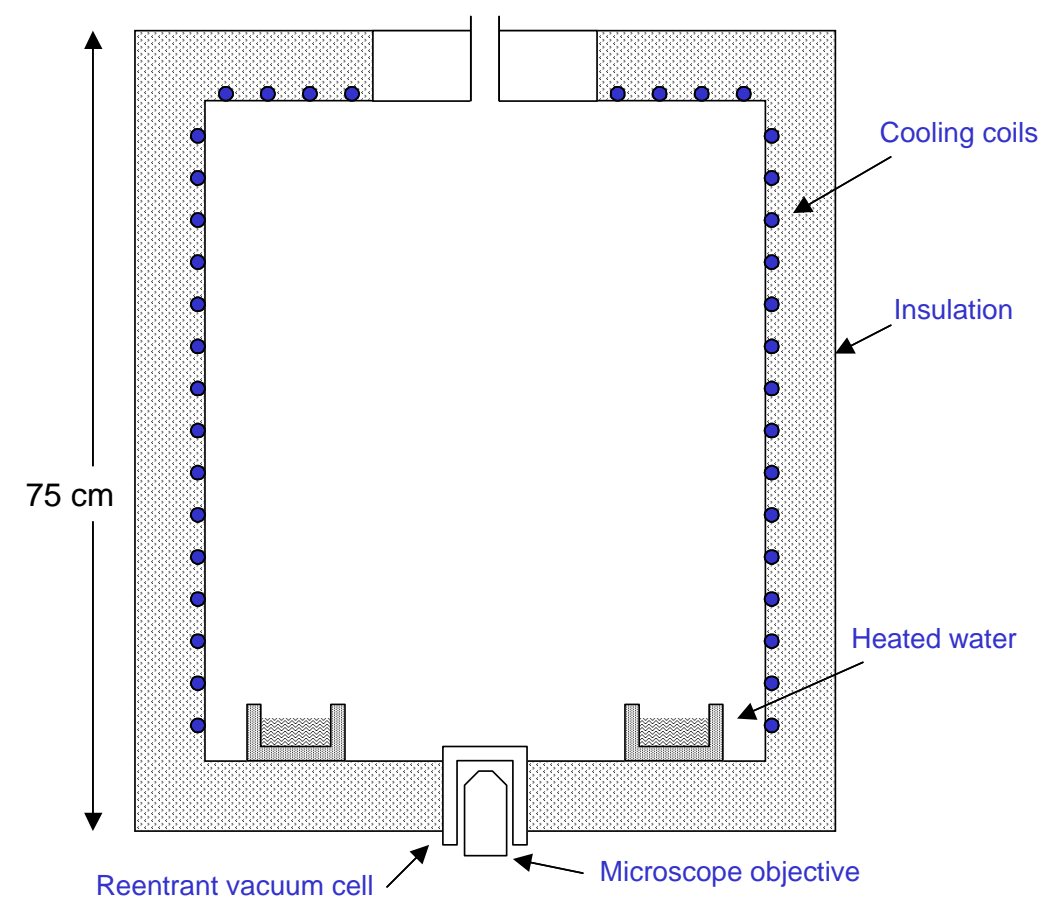

Figure 1. Schematic diagram of the chamber used for growing ice crystals in free-fall. Cooling coils refrigerate the chamber, while heated water supersaturates the air. Mixing via convection maintains a uniform air temperature within the chamber. A fleck of dry ice is dropped into the top of the chamber to nucleate ice crystals. The crystals grow while falling in the chamber, and some land on a window where they can be observed with a microscope. 


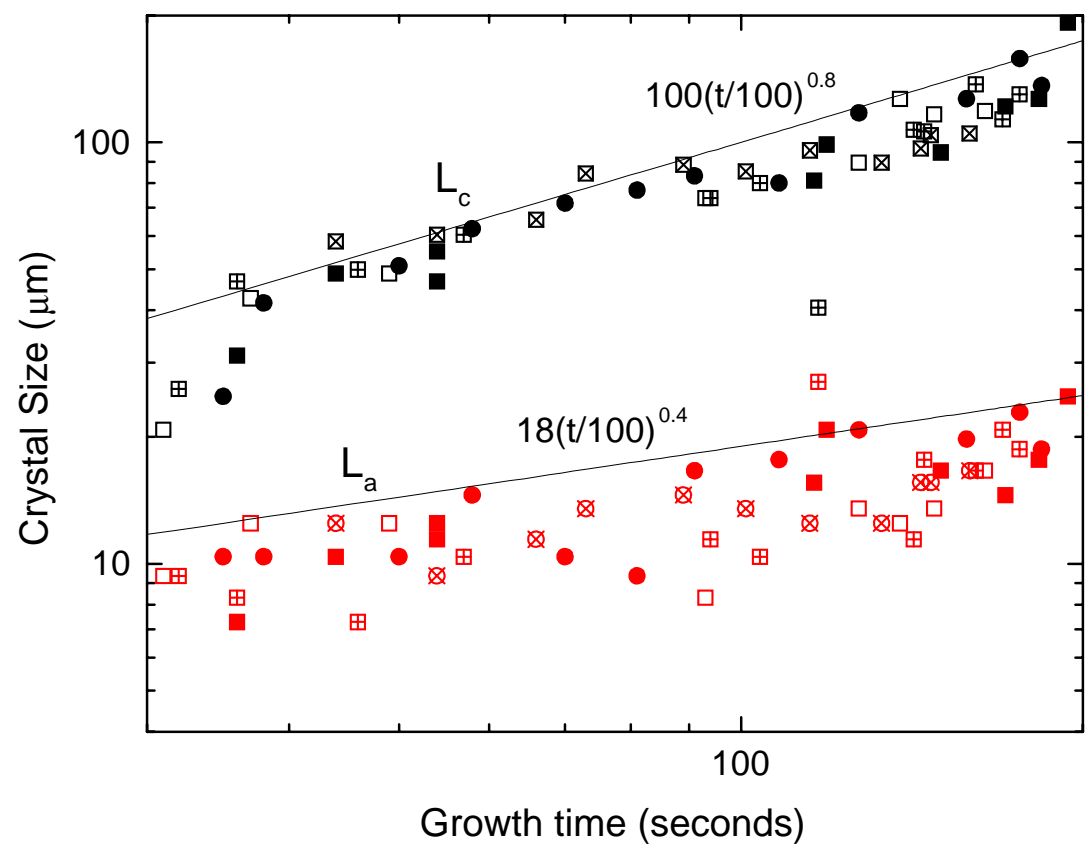

Figure 2. Measurements of the sizes of individual ice crystals as a function of time after nucleation, taken at an ambient supersaturation of 2.95 percent. Different point symbols represent different growth runs. Only the larger crystals were recorded at a given time, in order to measure the upper cut-off of the size distribution function. The lines indicate the approximate maximum crystal size as a function of time, representing crystal growth near the center of the growth chamber. 


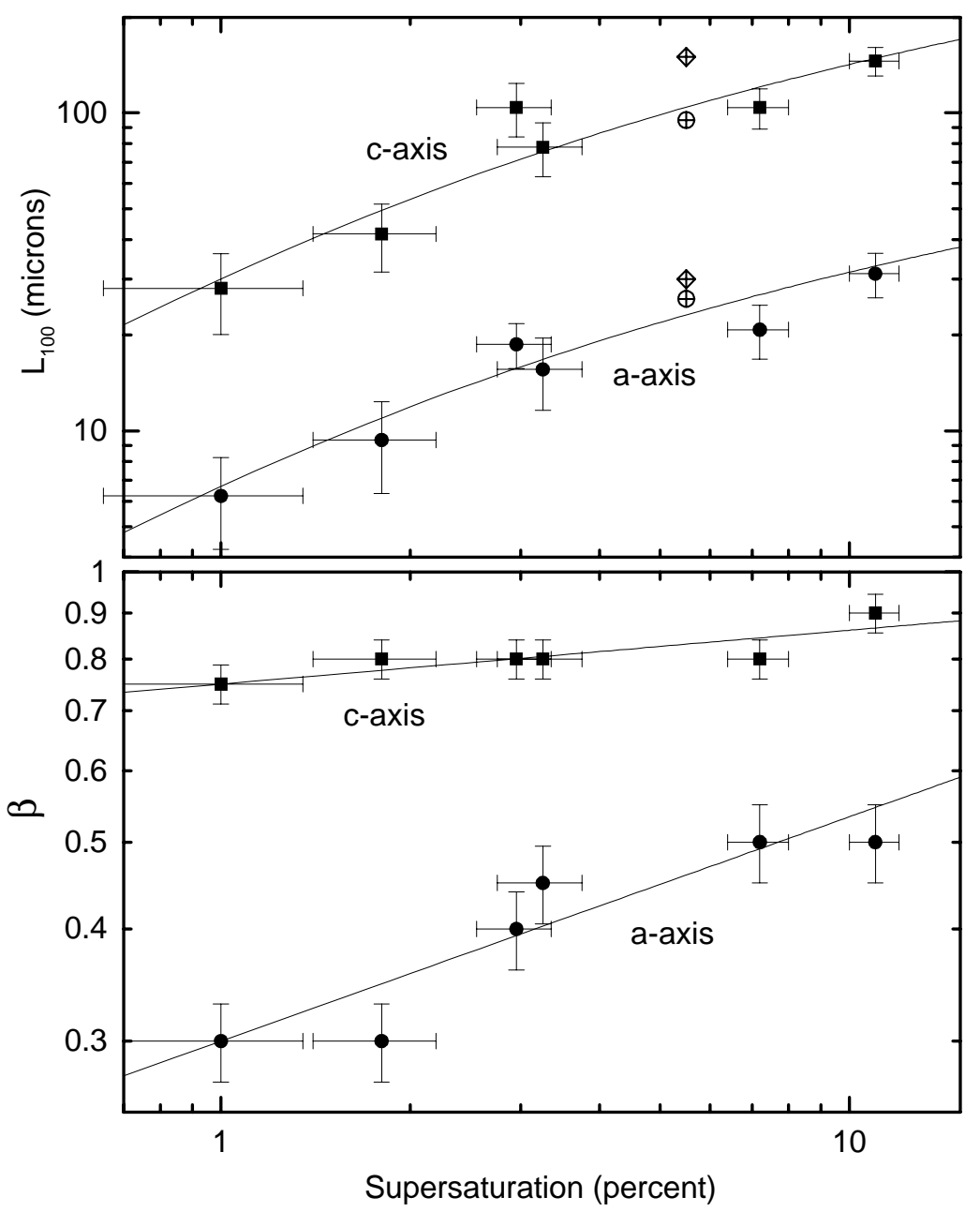

Figure 3. Fit parameters derived from growth data taken at different supersaturation levels, as described in the text. The open diamonds and circles show data by Ryan et. al [22] and Yamashita [21], respectively, taken at the water supersaturation level. 


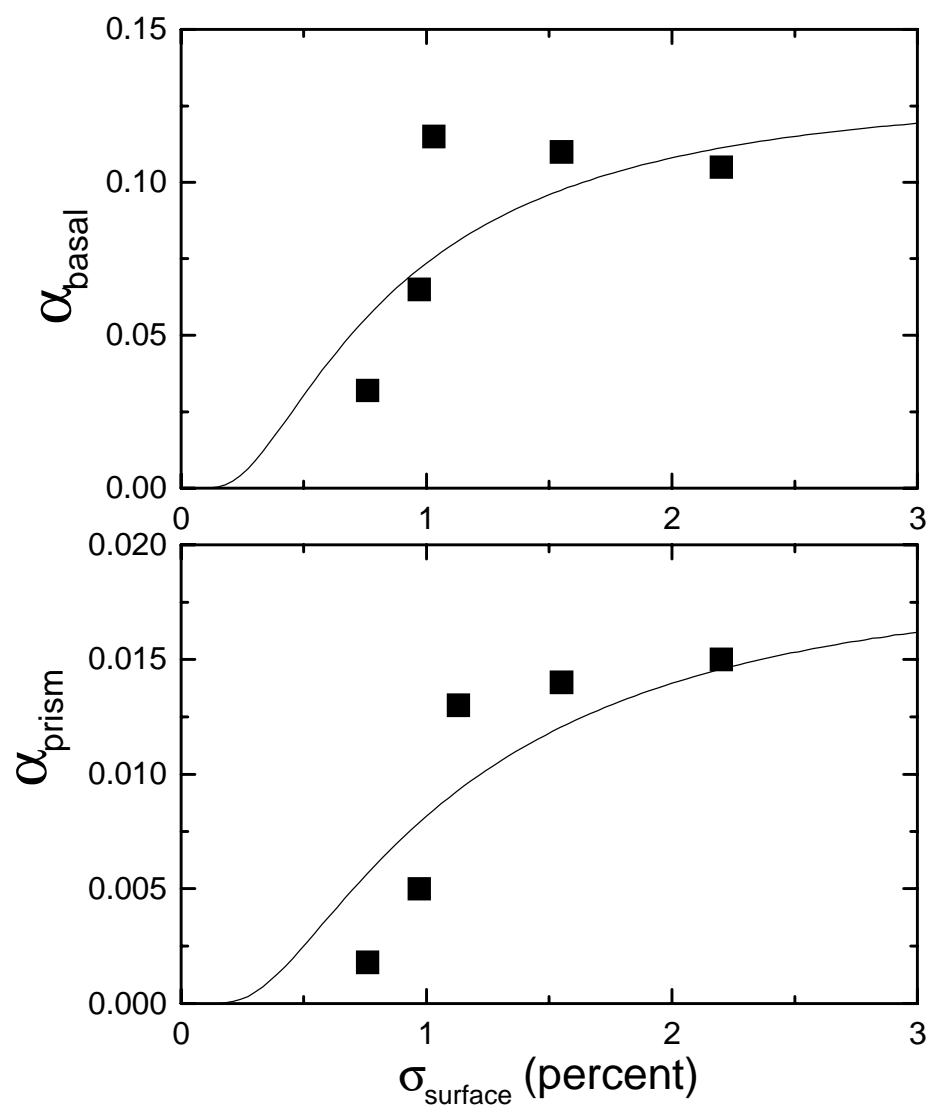

Figure 4. Inferred values of the condensation coefficients describing growth of the basal and prism faces of ice, as a function of supersaturation at the crystal surface. The curves show two-parameter fits to the data using the multinucleation model described in the text. 